\title{
STRUCTURE-PRESERVING ALGORITHMS FOR SIMPLE SLIDING CONTACT CONSTRAINT IN DIRECTOR-BASED GEOMETRIC EXACT BEAM
}

\author{
Jiawen Guo $^{1}$, Peter Betsch ${ }^{2}$ and Yue Zhang $^{3}$ \\ ${ }^{1}$ Institut für Mechanik, Karlsruhe Institute of Technology \\ Otto-Ammann-Platz 9, D-76131 Karlsruhe, Germany \\ jiawen.guo@partner.kit.edu \\ ${ }^{2}$ Institut für Mechanik, Karlsruhe Institute of Technology \\ Otto-Ammann-Platz 9, D-76131 Karlsruhe, Germany \\ peter.betsch@kit.edu, http://www.ifm.kit.edu/14_4655.php \\ ${ }^{3}$ Xidian University, 266 Xinglong Section of Xifeng Road \\ Xi' an, Shaanxi, 710126 China \\ zhangyue@xidian.edu.cn
}

Key words: Structure-preserving Method, Geometric Exact Beam Formulation, Sliding Contact Constraints, Large Flexibility

\begin{abstract}
Structure-preserving algorithms exhibit superior long-run numerical stability in nonlinear solid and elasto-multibody dynamics. This paper provides time integrators for large flexible dynamic systems combining the carrier-sliding contact pair between two beams. The time integrators maintain some of the structural characteristics, which include the momentum, energy, symplecity et al. In research of the beam modeling, the director-based geometrically exact beam formulation has been compared with the three-dimensional absolute nodal coordinate beam formulation, which is also widely used in dynamic modeling of slender structures. The sliding contact transition between adjacent elements on the sliding line has been finely considered to keep the continuity of the sliding contact. The structurepreserving method has been embedded into the numerical solvers for dynamic analysis. The advantage of the structure-preserving methods over the time-decaying methods on energy and momentum preserving properties has been demonstrated in the dynamic analysis for the flexible beams that undergo sliding contact.
\end{abstract}

\section{INTRODUCTION}

The situation where two bodies are hinged together and relative sliding occurs on the constraint surface is commonly used in slender suspension-cable system or flexible deployable structure. One of the difficulties in dynamic modeling refers to the description of the large rotation degrees of freedom in relative motion, especially when the contact relationship not only relies on the rigid modes of connection points but also the overall configuration in the deformed state. In the sliding contact pair, the contact node on the slave element is fixed, for example, on one tip, while the contact node can be located anywhere on 
the sliding line of the master element. Therefore, the sliding constraint is closely related to the dynamic description of the flexible body.

An efficient formulation for the large deformable finite element is based on the non-angle-based parameters in the director field $[1,2]$. The director-based formulation leads to an objective and path-independent orientation description, with which the design of numerical integration schemes for large displacements or strain problems is allowed to inherit the unconditional stability from linear regime.

Traditional numerical integration algorithms usually ignore the physical characteristics inherited from the continuous system. The numerical results distort the practical motion with the accumulation of numerical errors. Structure-preserving methods preserve some of the invariant geometric structures by identifying the corresponding properties such as the energy, momentum or symplectic form in the designing of the integrators. Gonzalez [7] introduced a definition of discrete directional derivative to construct the second-order conserving integration schemes, which lays the foundation of energy-momentum conserving algorithms used in nonlinear finite element analysis. Gonzalez [8] applied this method to elastodynamics for compressible and incompressible models. Muñoz and Jelenić [9] developed the incrementally based conserving algorithms for sliding joints modeled with the three-dimensional geometrically exact beams and analysed the preservation of energy and momentum. Leyendecker et al. [10] derived the energy-consistent time integration combined with the discrete null space size reduction method for semi-discrete nonlinear equations of motion. Romero [11] proved the possibility of constructing an infinite number of consistent and conserving methods by means of choosing the appropriate stress algorithmic definition for hyper-elastic materials. Due to the fact that conserving algorithms show

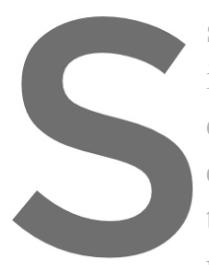
superior performance in nonlinear dynamic analysis, they have been
involving rigid body, slender cable/beam, shell, membrane and so
development of energy-momentum algorithms, which are systematic
chanical systems. Gebhardt et al. [13] put forward an object-orien
the dynamics of complex composite materials with a robust energy preserving method. Orden [14] discussed the symmetry-preserving con
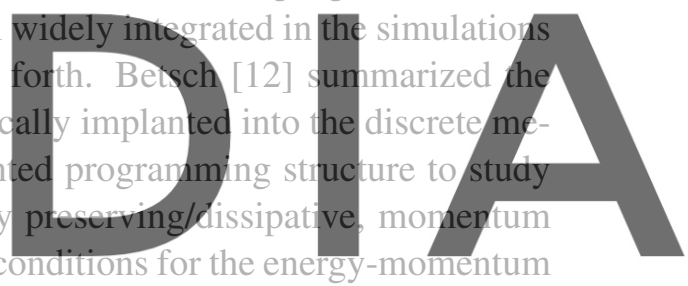
schemes designed on the basis of several expressions for discrete derivatives.

Register for free at https//www.scipedia.com to download the version without the watermark In this work, the aim is to improve the accuracy and stability of the numerical simulations for the con-

strained large flexible multi-body system. Considering the simple sliding contact joint, the typical configuration is established. We derive the director-based geometrically exact beam interpolated with quadratic B-spline in geometry to describe the flexible body system. we extend the energy-momentum time integration to the sliding joint constraint system with the involvement of the additional non-generalized intermediate coordinate.

\section{DIRECTOR-BASED GEOMETRICALLY EXACT BEAM FORMULATION WITH SMALL STRAIN MEASURE}

We first consider the kinematics of the geometrically exact beam with rectangular cross-section in director fields. The orientation of the beam cross-section is represented by the rotationless triad vectors. The triad vectors that are attached to the centroid of cross-section are denoted as $\left(\boldsymbol{D}_{1}, \boldsymbol{D}_{2}, \boldsymbol{D}_{3}\right) \in \mathfrak{R}^{3}$ in the reference configuration and $\left(\boldsymbol{d}_{1}, \boldsymbol{d}_{2}, \boldsymbol{d}_{3}\right) \in \mathfrak{R}^{3}$ in the current configuration according to Fig. 1 . The components along every triad compose the local coordinate system $\Theta=\left(\theta^{1}, \theta^{2}, \theta^{3}\right) \in \mathfrak{R}^{3}$ for the ma- 


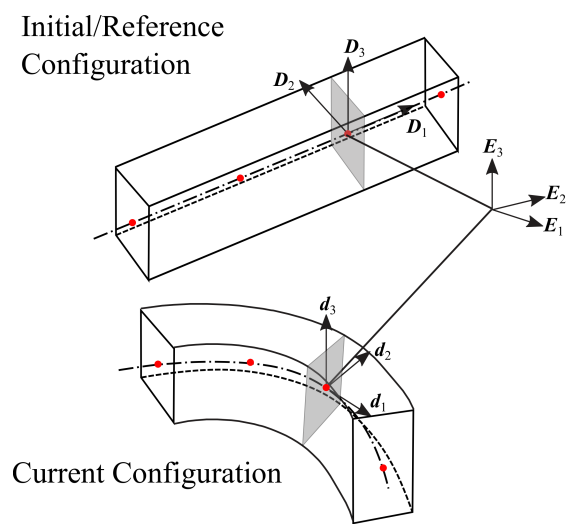

Figure 1: Beam configuration in different deformed states

terial point in beam. The set of the director triads are mutually orthonormal and can be related to the space-fixed inertial Cartesian frame $\left(\boldsymbol{E}_{1}, \boldsymbol{E}_{2}, \boldsymbol{E}_{3}\right) \in \mathfrak{R}^{3}$ by the rotation tensor $\boldsymbol{R}$

$$
\boldsymbol{d}_{I}=\boldsymbol{R}(s, t) \boldsymbol{E}_{I}, \quad \boldsymbol{R}=\boldsymbol{d}_{I} \otimes \boldsymbol{E}_{I}
$$

In order to maintain the cross-section rigidity, the mutual orthonormality is obtained by introducing the six independent internal constraints
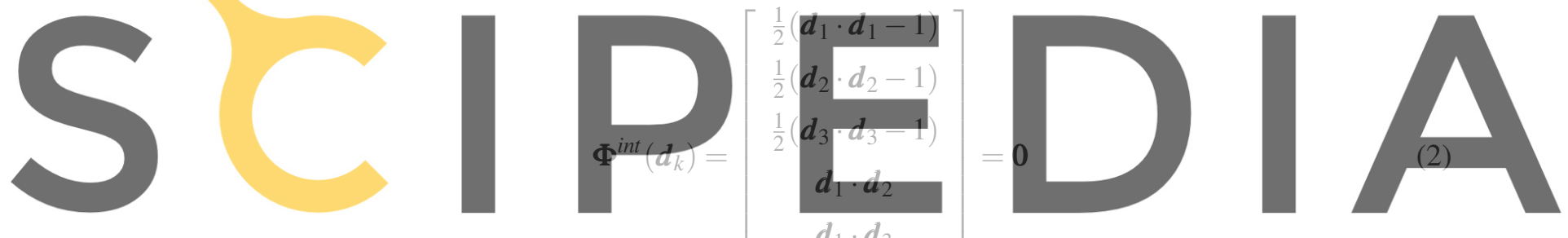

Register for free at https//www.scipedia.com to dovibloagl the version without the watermark

The position vector of the material point in the current deformed configuration is given as follows

$$
\boldsymbol{x}\left(\theta^{\alpha}, s, t\right)=\boldsymbol{\varphi}(s, t)+\theta^{\alpha} \boldsymbol{d}_{\alpha}(s, t)
$$

where $\alpha=2,3$ are the indexes of the material coordinates on the cross-section and $\boldsymbol{\varphi}(s, t)$ represents the space curve of centroids. The material coordinates do not change with the rigid motion and deformation.

We then derive the deformation gradient using the partial derivatives of position vectors in deformed configuration with respect to those in undeformed configuration and the gradient tensor can be decomposed as

$$
\boldsymbol{F}=\frac{\partial \boldsymbol{x}}{\partial \boldsymbol{X}}=\frac{\partial \boldsymbol{x}}{\partial \boldsymbol{\theta}}\left[\frac{\partial \boldsymbol{X}}{\partial \boldsymbol{\theta}}\right]^{-1}
$$

where the Jacobian matrix $\frac{\partial \boldsymbol{X}}{\partial \boldsymbol{\theta}}$ is the orthogonal matrix. Substituting Equ. (3) into Equ. (4), the deformation gradient is rewritten as

$$
\boldsymbol{F}=\left(\boldsymbol{\varphi}_{, s}+\theta^{\alpha} \boldsymbol{d}_{\alpha, s}-\boldsymbol{d}_{1}\right) \otimes \boldsymbol{D}_{1}+\boldsymbol{d}_{I} \otimes \boldsymbol{D}_{I}
$$


where the pure stretch vector can be extracted from the first term in Equ. (5) as

$$
\boldsymbol{a}=\boldsymbol{\varphi}_{, s}+\theta^{\alpha} \boldsymbol{d}_{\alpha, s}-\boldsymbol{d}_{1}
$$

In this research, we are interested in developing the consistent constitutive law for the large deformation in three-dimensional continuum. As the linear elastic constitutive law is subjected to the small strain assumption, the large deformation will be transformed to a small strain description. It is achieved by eliminating some of the higher-order nonlinear terms in the strain expression. This strain approximation is based on the commonly used Green-Lagrange strain tensor in the standard form

$$
\boldsymbol{E}=\frac{1}{2}\left(\boldsymbol{F}^{T} \boldsymbol{F}-\boldsymbol{I}\right)
$$

Inserting Equ. (5) and Equ. (6) into Equ. (7), the Green-Lagrange strain tensor is expanded in detail as

$$
\boldsymbol{E}=\frac{1}{2}\left[(\boldsymbol{a} \cdot \boldsymbol{a}) \boldsymbol{D}_{1} \otimes \boldsymbol{D}_{1}+2\left(\boldsymbol{a} \cdot \boldsymbol{d}_{I}\right) \boldsymbol{D}_{I} \otimes \boldsymbol{D}_{1}\right]
$$

The large strain tensor given in matrix form is

$$
E=\frac{1}{2}\left[\begin{array}{ccc}
(a \cdot a)+2 a \cdot d_{1} & a \cdot d_{2} & a \cdot d_{3} \\
a \cdot d_{2} & 0 & 0 \\
a \cdot d_{3} & 0 & 0
\end{array}\right]
$$

In order to make the strain tensor compatible with the consistent constitutive formulation, the highet

order terms expanded firon
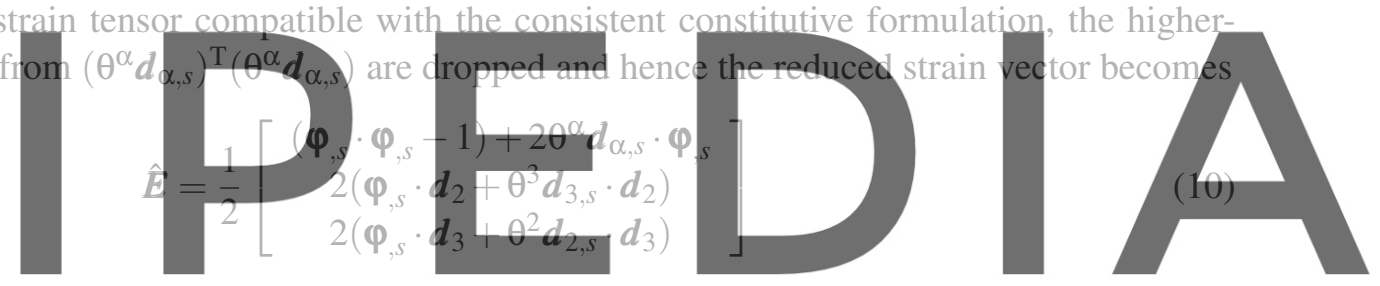

From a physical standpoint, the small strain vector $\hat{E}$ could be transformed to the generalized strain

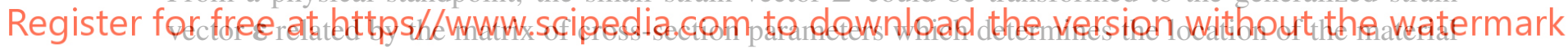

point. The transformation reads

$$
\hat{E}=\hat{D} \mathcal{C}
$$

where the transformation matrix is

$$
\hat{\boldsymbol{D}}=\left[\begin{array}{cccccc}
1 & 0 & 0 & 0 & \theta^{3} & \theta^{2} \\
0 & 1 & 0 & -\theta^{3} & 0 & 0 \\
0 & 0 & 1 & \theta^{2} & 0 & 0
\end{array}\right]
$$

and the generalized strain vector $\boldsymbol{\varepsilon}$ contains the strain components with material description which specifies the axial, shear, torsional and bending strain in the following expressions

$$
\boldsymbol{\varepsilon}=\left[\begin{array}{c}
\boldsymbol{\varepsilon} \\
\gamma_{2} \\
\gamma_{3} \\
\kappa_{1} \\
\kappa_{2} \\
\kappa_{3}
\end{array}\right]=\left[\begin{array}{c}
\frac{1}{2}\left(\boldsymbol{\varphi}_{, s} \boldsymbol{\varphi}_{, s}-1\right) \\
\boldsymbol{\varphi}_{, s} \cdot \boldsymbol{d}_{2} \\
\boldsymbol{\varphi}_{, s} \cdot \boldsymbol{d}_{3} \\
\boldsymbol{d}_{2, s} \cdot \boldsymbol{d}_{3} \\
\boldsymbol{\varphi}_{, s} \cdot \boldsymbol{d}_{3, s} \\
\boldsymbol{\varphi}_{, s} \cdot \boldsymbol{d}_{2, s}
\end{array}\right]
$$




\section{SIMPLE SLIDING JOINT CONSTRAINT}

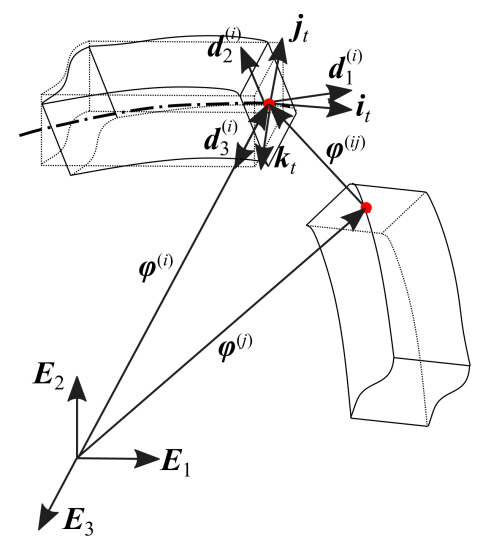

Figure 2: Kinematics of the sliding joint

Fig. 2 shows the kinematics of the sliding joint between the two connection points respectively on the very flexible beam $i$ and $j$. The simple sliding joint is similar to the local spherical joint with the relative displacements restricted by three position constraint equations, while the relative rotations are allowed in three-dimensional space. The vector $\varphi^{i}$ denotes the global position of the contact point on the twoend-fixed beam $i$, and its position on the beam center line is decided by the arc-length parameter $s^{i}$. The contact point on the one-end-free beam $j$ is selected at the tip where the $\varphi^{j}$ denotes the global tip position. constraint equations
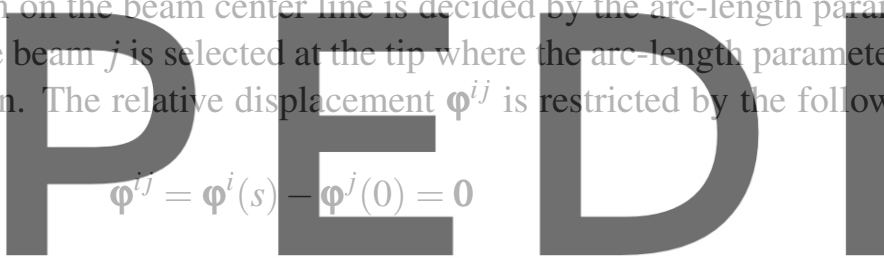

For smoothly spatial discretization, the approximation of the position of any point on the beam centerline Register fon fre calculated with basis functionsin the If A intergolation the version without the watermark

$$
\varphi^{\mathrm{h}}(s, t)=\sum_{A=1}^{n e} N^{A}(s) \varphi^{A}(t)
$$

where in this research, $\boldsymbol{N}^{A}(s)$ are obtained by the quadratic B-spline curve and are analogous to the shape function used in the finite element methods. $\varphi^{A}(t)$ are the control points on position level. Similarly the approximated director triads are expressed by

$$
\boldsymbol{d}_{k}^{\mathrm{h}}(s, t)=\sum_{A=1}^{n e} \boldsymbol{N}^{A}(s) \boldsymbol{d}_{k}^{\mathrm{A}}(t), \quad k=1,2,3
$$

With the B-spline geometry description, the internal control points do not always lie on the practical space curve. The joint positions in Equ. (14) are replaced by the discrete version of position vectors in Equ. (15). Accordingly, the kinematic constraints relating to the control points on sliding elements are re-written as

$$
\boldsymbol{\varphi}^{i j \mathrm{~h}}=\sum_{A=1}^{n e^{i}} \boldsymbol{N}^{i A}(s) \boldsymbol{\varphi}^{i A}(t)-\sum_{A=1}^{n e^{j}} \boldsymbol{N}^{j A}(0) \boldsymbol{\varphi}^{j A}(t)=\mathbf{0}
$$


Then, the virtual work of the inertia force for the semi-discrete model is given by

$$
G_{d y n}^{\mathrm{h}}\left(\boldsymbol{q}^{\mathrm{h}}, \delta \boldsymbol{q}^{\mathrm{h}}\right)=\int_{V_{0}} \sum_{A, B=1}^{n e} \rho \delta \boldsymbol{q}^{A^{\mathrm{T}}} N^{A} N^{B} \tilde{\boldsymbol{M}}^{A B} \ddot{\boldsymbol{q}}^{B} d V
$$

and the virtual work done by the internal force is given by

$$
G_{i n t}^{\mathrm{h}}\left(\boldsymbol{q}^{\mathrm{h}}, \delta \boldsymbol{q}^{\mathrm{h}}\right)=\int_{V_{0}} \delta \hat{\boldsymbol{E}}^{\mathrm{h}} \cdot \hat{\boldsymbol{S}}^{\mathrm{h}} d V
$$

The complete virtual work of constraint forces includes the following two parts

$$
G_{\lambda}^{\mathrm{h}}\left(\left\{\boldsymbol{q}^{\mathrm{h}}, s, \boldsymbol{\lambda}\right\},\left\{\delta \boldsymbol{q}^{\mathrm{h}}, \delta s, \delta \boldsymbol{\lambda}\right\}\right)=G_{\lambda}^{i n t, \mathrm{~h}}+G_{\lambda}^{s, \mathrm{~h}}
$$

where

and

$$
G_{\lambda}^{i n t, \mathrm{~h}}=\sum_{A=1}^{n e} \delta \lambda^{i n t, A} \cdot \boldsymbol{\Phi}^{i n t, A}\left(\boldsymbol{d}_{k}^{A}\right)+\sum_{A=1}^{n e} \delta \boldsymbol{d}_{k}^{A} \cdot \boldsymbol{\Phi}_{d_{k}}^{i n t, A \mathrm{~T}} \lambda^{i n t, A}
$$

$$
G_{\lambda}^{s, \mathrm{~h}}=\delta \lambda^{s} \cdot \boldsymbol{\Phi}^{s}\left(\boldsymbol{q}^{A}, \boldsymbol{q}^{B}, s\right)+\delta \boldsymbol{q}^{A} \cdot \boldsymbol{\Phi}_{q^{A}}^{s \mathrm{~T}} \boldsymbol{\lambda}^{s, A}+\delta \boldsymbol{q}^{B} \cdot \boldsymbol{\Phi}_{\boldsymbol{q}^{B}}^{s \mathrm{~T}} \boldsymbol{\lambda}^{s, B}+\delta s \cdot \boldsymbol{\Phi}_{s}^{s \mathrm{~T}} \boldsymbol{\lambda}^{s, s}
$$

The equilibrium of the discrete weak form for the constrained equations of motion reads
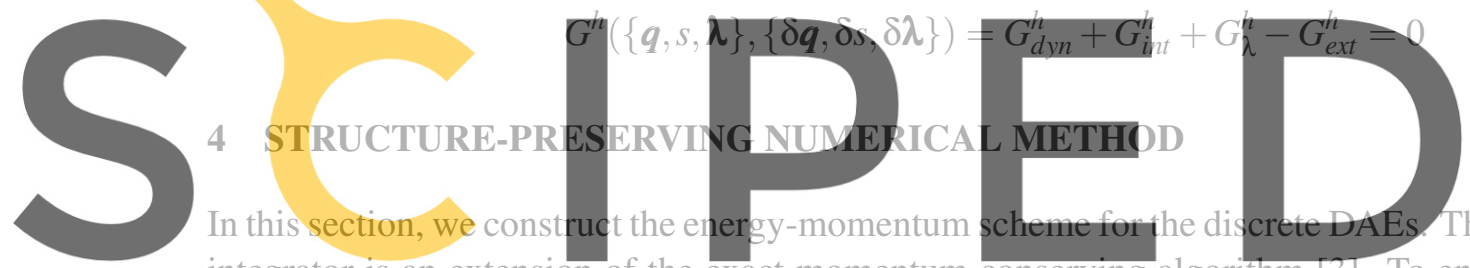

integrator is an extension of the exact momentum conserving algorithm [3]. To ensure that the numerical integration performs reliably in long-term time stepping, the system energy is controlled to be a Register fernfreedatrbttess//www.scipedia.com to download the version without the watermark

In the state space, the position and velocity coordinates are approximated according to the midpoint rule which conserves the generalized momenta along the solution trajectory. For both the generalized and non-generalized intermediate coordinates, the predictions for the next step is expanded through the average values on velocity level as

$$
\begin{aligned}
& \boldsymbol{q}_{n+1}-\boldsymbol{q}_{n} \approx h \dot{\boldsymbol{q}}_{n+\frac{1}{2}}=\frac{h}{2}\left(\dot{\boldsymbol{q}}_{n+1}+\dot{\boldsymbol{q}}_{n}\right) \\
& \boldsymbol{s}_{n+1}-\boldsymbol{s}_{n} \approx h \dot{\boldsymbol{s}}_{n+\frac{1}{2}}=\frac{h}{2}\left(\dot{\boldsymbol{s}}_{n+1}+\dot{\boldsymbol{s}}_{n}\right)
\end{aligned}
$$

And similarly, the velocity coordinates are approximated by the average accelerations

$$
\dot{\boldsymbol{q}}_{n+1} \approx \dot{\boldsymbol{q}}_{n}+h \ddot{\boldsymbol{q}}_{n+\frac{1}{2}}, \quad \dot{\boldsymbol{s}}_{n+1} \approx \dot{\boldsymbol{s}}_{n}+h \ddot{\boldsymbol{s}}_{n+\frac{1}{2}}
$$


The average accelerations correspond to the resultant generalized forces at the mid-time configuration. Inserting the approximated kinematic variables from Equ. (24)-(25) into the dynamic equations, the discrete numerical model with additional constrained force equations are given by

$$
\begin{aligned}
& \boldsymbol{M}\left(\dot{\boldsymbol{q}}_{n+1}-\dot{\boldsymbol{q}}_{n}\right)=\left.h\left(\boldsymbol{Q}_{a}-\nabla_{\boldsymbol{q}} U_{e}\right)\right|_{n+\frac{1}{2}}-\left.h \boldsymbol{\Phi}_{\boldsymbol{q}}^{\mathrm{T}}\right|_{n+\frac{1}{2}} \boldsymbol{\lambda}_{n+1} \\
& \left.\boldsymbol{\Phi}_{\boldsymbol{s}}^{\mathrm{T}}\right|_{n+\frac{1}{2}} \boldsymbol{\lambda}_{n+1}=\mathbf{0} \\
& \boldsymbol{\Phi}\left(\boldsymbol{q}_{n+1}, \boldsymbol{s}_{n+1}\right)=\mathbf{0}
\end{aligned}
$$

As for the midpoint rule, the mid-time generalized forces can be obtained from the standard gradients of energy functions, for example $\nabla_{q} U_{e}\left(\boldsymbol{q}_{n+\frac{1}{2}}\right)$. However, in the energy-momentum scheme (Equ. (26)), the mid-time gradient $\left.\nabla_{\boldsymbol{q}} U_{e}\right|_{n+\frac{1}{2}}$ is approximated by the discrete gradient $\bar{\nabla}_{\boldsymbol{q}} U_{e}\left(\boldsymbol{q}_{n}, \boldsymbol{q}_{n+1}\right)$ expanded as follows

$$
\left.\nabla_{q} U_{e}\right|_{n+\frac{1}{2}}=\bar{\nabla}_{q} U_{e}\left(q_{n}, q_{n+1}\right)=\frac{\partial \boldsymbol{\varepsilon}^{\mathrm{T}}}{\partial q}\left(q_{n+\frac{1}{2}}\right) \mathrm{D} U_{e}\left(\varepsilon_{n+\frac{1}{2}}\right)
$$

with the average strain measure $\boldsymbol{\varepsilon}_{n+\frac{1}{2}}=\frac{1}{2}\left(\boldsymbol{\varepsilon}_{n}+\boldsymbol{\varepsilon}_{n+1}\right) \neq \boldsymbol{\varepsilon}\left(\boldsymbol{q}_{n+\frac{1}{2}}\right)$.

The specific energy contributed by the constraint enforcement is defined as $\boldsymbol{\lambda} \cdot \boldsymbol{\Phi}(\boldsymbol{q}, \boldsymbol{s})$ using the Lagrange multiplier method. Instead of being treated as the system variables, the discrete Lagrange multipliers $\boldsymbol{\lambda}_{n+1}$ can be directly solved from the Newton iterations. Then, the approximation of the mid-time constrained forces are obtained from the discrete derivatives of the constrain equations respectively relative to the generalized coordin
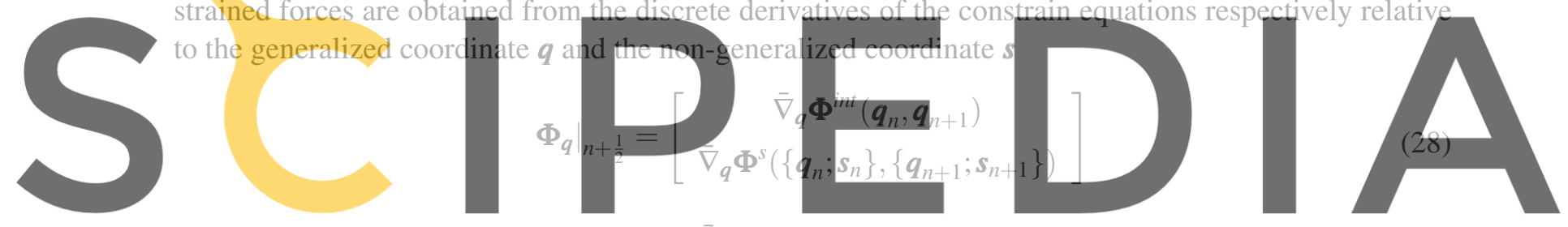

$$
\left.\Phi_{s}\right|_{n+\frac{1}{2}}=\bar{\nabla}_{s} \Phi^{s}\left(\left\{q_{n} ; s_{n}\right\},\left\{q_{n+1} ; s_{n+1}\right\}\right)
$$

(29)

Register for free at https//www.scipedia.com to download the version without the watermark where the Jacobian matrix $\bar{\nabla}_{q} \Phi^{\text {int }}=\mathrm{D}_{q} \Phi^{\text {int }}, \bar{\nabla}_{q} \Phi^{s}=\mathrm{D}_{q} \Phi^{s}$ and $\bar{\nabla}_{s} \Phi^{s}=\mathrm{D}_{s} \Phi^{s}$ are

$$
\begin{aligned}
& \mathrm{D}_{\boldsymbol{q}} \boldsymbol{\Phi}^{i n t, A}\left(\boldsymbol{q}_{n+\frac{1}{2}}\right)=\left[\begin{array}{cccc}
\mathbf{0}_{1 \times 3} & \boldsymbol{d}_{1\left(n+\frac{1}{2}\right)}^{A \mathrm{~T}} & \ldots & \ldots \\
\mathbf{0}_{1 \times 3} & \ldots & \boldsymbol{d}_{2\left(n+\frac{1}{2}\right)}^{A \mathrm{~T}} & \ldots \\
\mathbf{0}_{1 \times 3} & \ldots & \ldots & \boldsymbol{d}_{3\left(n+\frac{1}{2}\right)}^{A \mathrm{~T}} \\
\mathbf{0}_{1 \times 3} & \boldsymbol{d}_{2\left(n+\frac{1}{2}\right)}^{A \mathrm{~T}} & \boldsymbol{d}_{1\left(n+\frac{1}{2}\right)}^{A \mathrm{~T}} & \ldots \\
\mathbf{0}_{1 \times 3} & \boldsymbol{d}_{3\left(n+\frac{1}{2}\right)}^{A \mathrm{~T}} & \ldots & \boldsymbol{d}_{1\left(n+\frac{1}{2}\right)}^{A \mathrm{~T}} \\
\mathbf{0}_{1 \times 3} & \ldots & \boldsymbol{d}_{3\left(n+\frac{1}{2}\right)}^{A \mathrm{~T}} & \boldsymbol{d}_{2\left(n+\frac{1}{2}\right)}^{A \mathrm{~T}}
\end{array}\right] \\
& \mathrm{D}_{\boldsymbol{q}} \boldsymbol{\Phi}^{s}\left(\left\{\boldsymbol{q}_{n+\frac{1}{2}} ; \boldsymbol{s}_{n+\frac{1}{2}}\right\}\right)=\left[\begin{array}{llllllllll}
\ldots & N^{A}\left(s_{n+\frac{1}{2}}\right) \boldsymbol{I}_{3} & \mathbf{0}_{3 \times 9} & \ldots & \mathbf{0}_{3 \times 1} & \ldots & -N^{B}(0) \boldsymbol{I}_{3} & \mathbf{0}_{3 \times 9} & \ldots
\end{array}\right] \\
& \mathrm{D}_{\boldsymbol{s}} \boldsymbol{\Phi}^{s}\left(\left\{\boldsymbol{q}_{n+\frac{1}{2}} ; \boldsymbol{s}_{n+\frac{1}{2}}\right\}\right)=\left[\sum_{A=1}^{n e^{i}} \boldsymbol{N}_{s}^{i A}\left(s_{n+\frac{1}{2}}\right) \boldsymbol{\varphi}_{n+\frac{1}{2}}^{i A}\right]
\end{aligned}
$$




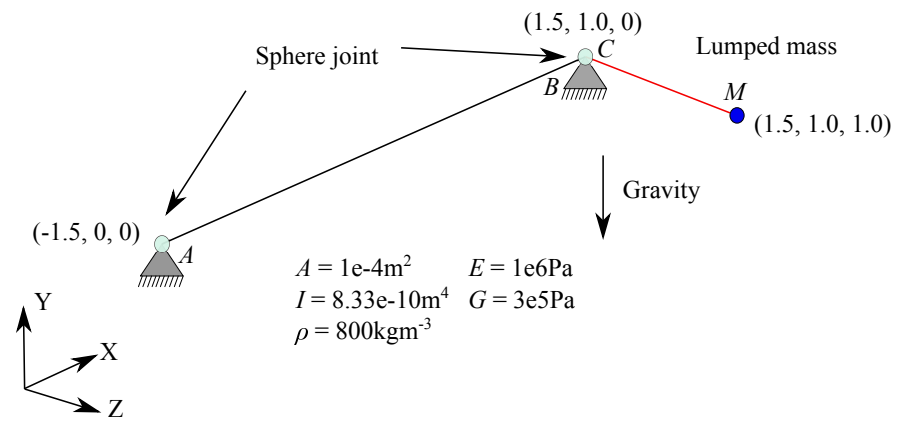

Figure 3: Initial configuration and parameters

\section{NUMERICAL ANALYSIS}

In the first example, the free falling case takes place in the three-dimensional space. The initial positions of the two beams and the structure and material parameters are listed in the Fig. (3). The beam AB is fixed into the space by the sphere joints at both ends. The sliding beam CM is parallel to axis $\mathrm{Z}$ and is undeformed in the initial configuration. The beam CM not only slides along AB but also swing in the perpendicular plane YZ. A lumped mass with $1 \mathrm{~kg}$ is attached to the free tip $\mathrm{M}$ and falls freely under the effect of gravity $\left(g=[0.0,-9.80665,0.0]^{\mathrm{T}}\right)$.

This is a typical case that was successively studied by Sugiyama et al. [4], Mũnoz and Jeleníc [5], Gerstmayr and Shabana [6] this case, the geometrically compared with the ANC momentum time integrat In Fig. (4), we carefully
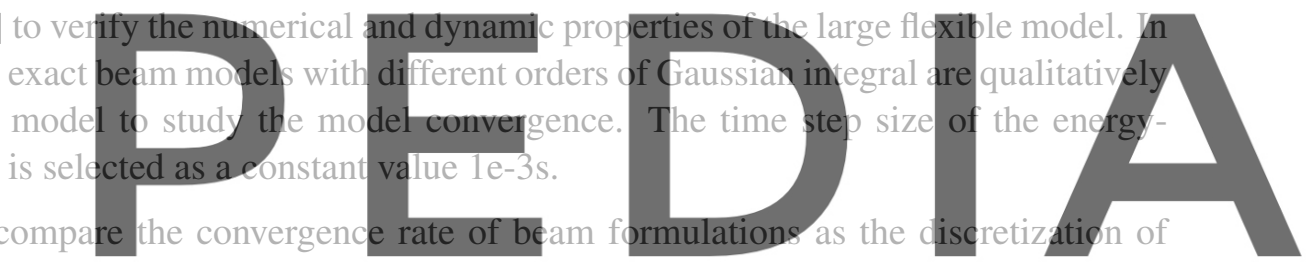
carrier beam continues to be refined. For reduced order beam elements, the overall trajectories of lumped

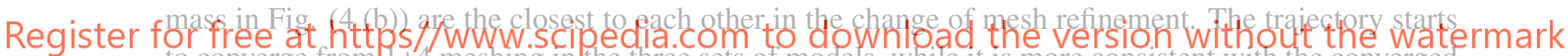
to converge from $1+4$ meshing in the three sets of models, while it is more consistent with the converged trajectory in the final stage in Fig. (4 (b)). Moreover, the tip lumped mass in geometrically exact beams swings back more at the end of converged trajectory compared with the absolute nodal coordinate beam, which reflects larger flexible deformation in the former converged models.

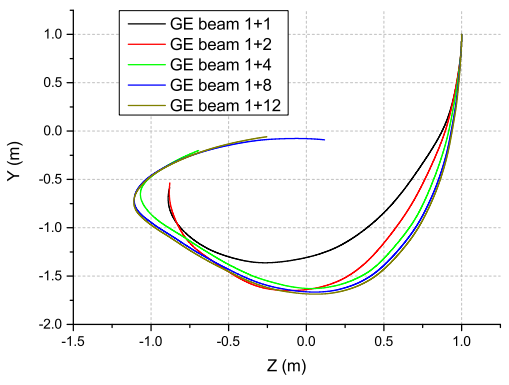

(a) Full order GE beam

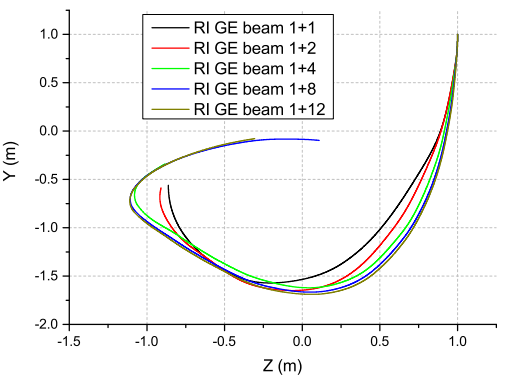

(b) Reduced order GE beam

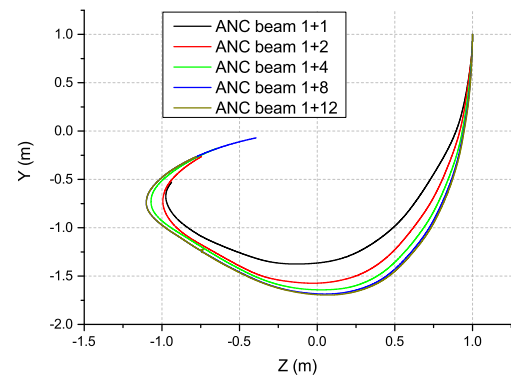

(c) ANC beam

Figure 4: Comparison of positions of lumped mass 


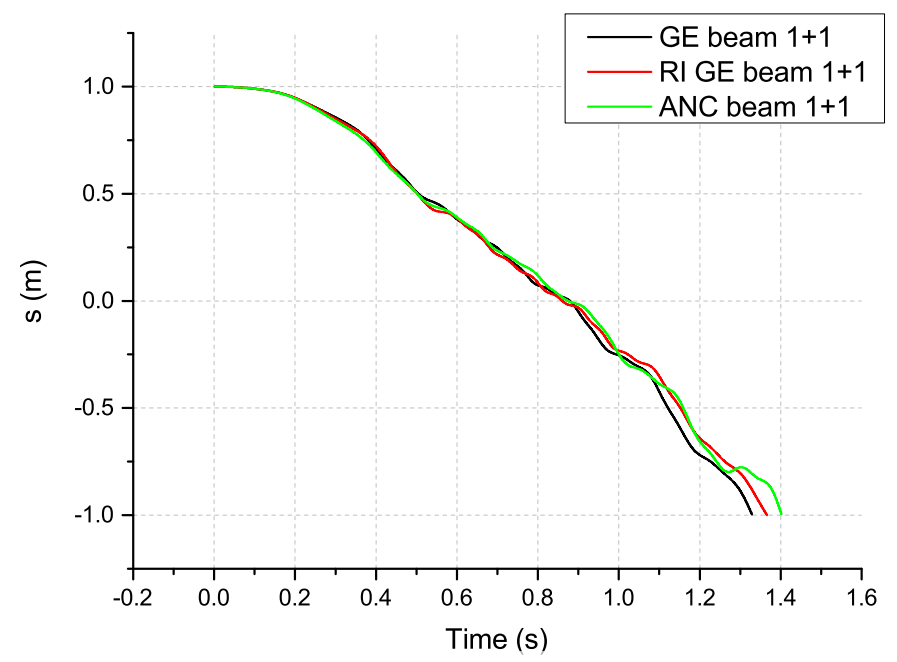

Figure 5: Comparison of positions of the lumped mass, $1+1$ meshing

The curves of sliding position along beam $\mathrm{AB}$ is shown in Fig. (5). With the $1+1$ meshing, the contact joint moves at different speeds. The sliding joint on the full order GE beam first reaches the bottom, which is consistent with the previous comparisons of large deformation.

Next, the numerical performance of the energy-momentum integrator is compared with the structural integrators and the mid-point rule. The time step sizes of all time integrators are set as $1 \mathrm{e}-3 \mathrm{~s}$ and the numerical dumping parameters in the structural integrators are $\alpha=-0$. (HHT) $\gamma=0.5, \beta=$
mark).

Fig. (6) displays the evolution of total energy in each case. The tota momentum integrator conserves the best in both meshing cases comp

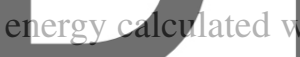
The energy blow-up observed in curves indicates the instability of the Newmark method and the HHT Register for free at https//www.scipedia.com to download the version without the watermark

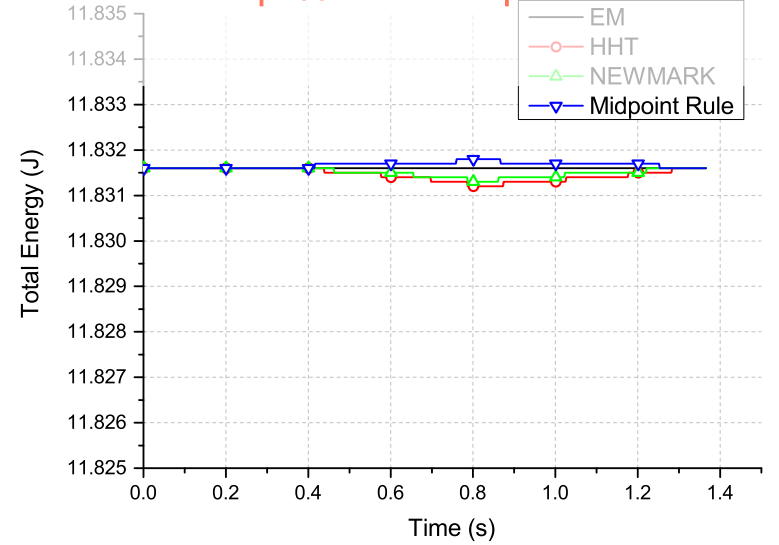

(a) $1+1$ meshing

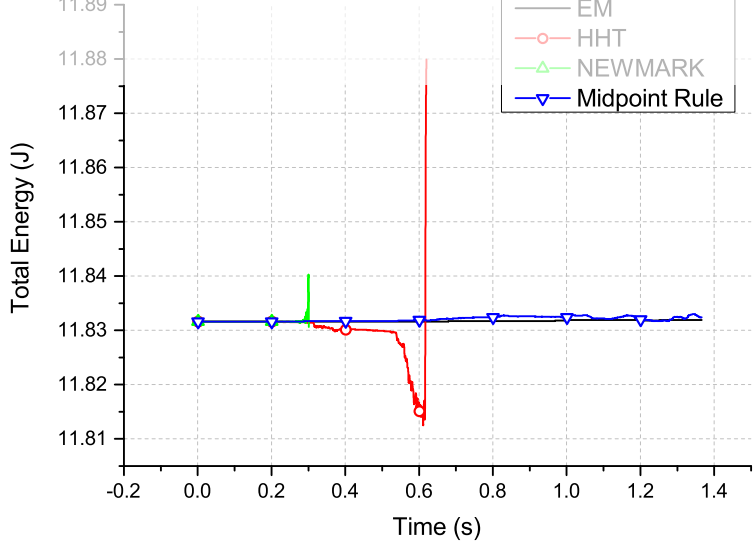

(b) 1+4 meshing

Figure 6: Total energy, $h=1 \mathrm{e}-3$ 
method for high-nonlinear problems. In the case of $1+1$ meshing, no contact transition occurs between elements. Therefore, the balance of the time-discrete equations of motion can be guaranteed as long as the iteration converges.

Once the beam is divided into multiple elements, contact transitions between the adjacent elements occurs. As the contact transition occurs in the $1+4$ meshing, the numerical equilibrium suffers from the discontinuities caused by the jumping between elements. But, even though the energy fluctuates more severely, the energy-momentum integrator and the mid-point rule show better robustness, while the Newmark and HHT integrator successively fail to converge at around $0.3 \mathrm{~s}$ and $0.6 \mathrm{~s}$. The conserving properties remain exactly conserved only when the sliding motion occurs within a single element. Besides, there exists the divergence of total energy using mid-point rule, while the energy-momentum integrator performs the best, even though the error of physical discretization cannot be resolved in the numerical model.

\section{CONCLUSIONS}

The nonlinear modeling and computation of the large deformable body with sliding joint constraint are proposed in this research. Based on the linear elastic theory, the large deformation is transformed to a small strain measure that can be compatible with the constitutive law. The director-based geometrically exact beam shows good convergence and efficiency with IGA description when the simple sliding constraint is implemented between the carrier beam and the sliding beam. Obviously, physical errors aggravate the accumulation of numerical errors and unconditional stability can not be ensured in the strong nonlinear cases. Compared with the structural integrators, the structure-preserving algorithms exhibit relatively superior numerical stability when the discontinuous contact transition occurs.

\section{ACIKNOWLEDGMENT}

This project was suppored by the China Scholarsh

ural Science Foundation of China (51975044).

Register for free at https//www.scipedia.com to download the version without the watermark

REFERENCES

[1] Romero, I. and Armero, F. An objective finite element approximation of the kinematics of geometrically exact rods and its use in the formulation of an energy-momentum conserving scheme in dynamics. International Journal for Numerical Methods in Engineering. (2002) 54(12):1683-1716.

[2] Leyendecker, S., Betsch, P. and Steinmann, P. Objective energy-momentum conserving integration for the constrained dynamics of geometrically exact beams. Computer Methods in Applied Mechanics and Engineering. (2006) 195(19-22):2313-2333.

[3] Simo, J. C., Tarnow, N. and Wong, K. Exact energy-momentum conserving algorithms and symplectic schemes for nonlinear dynamics. Computer methods in applied mechanics and engineering. (1992) 100(1): 63-116.

[4] Sugiyama, H., Escalona, J. L. and Shabana, A. A. Formulation of three-dimensional joint constraints using the absolute nodal coordinates. Nonlinear Dynamics. (2003) 31(2): 167-195.

[5] Muñoz, J. J. and Jelenić, G. Sliding contact conditions using the master-slave approach with ap- 
plication on geometrically non-linear beams. International journal of solids and structures. (2004) 41(24-25): 6963-6992.

[6] Gerstmayr, J. and Shabana, A. A. Analysis of thin beams and cables using the absolute nodal coordinate formulation. Nonlinear Dynamics. (2006) 45(1-2): 109-130.

[7] Gonzalez, O. Time integration and discrete Hamiltonian systems. Journal of Nonlinear Science. (1996) 6(5): 449-467.

[8] Gonzalez, O. Exact energy and momentum conserving algorithms for general models in nonlinear elasticity. Computer Methods in Applied Mechanics and Engineering. (2000) 190(13-14): 17631783.

[9] Muñoz, J. J. and Jelenić, G. Sliding joints in 3D beams: conserving algorithms using the masterslave approach. Multibody System Dynamics. (2006) 16(3): 237-261.

[10] Leyendecker, S., Betsch, P. and Steinmann, P. The discrete null space method for the energyconsistent integration of constrained mechanical systems. Part III: Flexible multibody dynamics. Multibody System Dynamics. (2008) 19(1): 45-72.

[11] Romero, I. An analysis of the stress formula for energy-momentum methods in nonlinear elastodynamics. Computational Mechanics. (2012) 50(5): 603-610.

[12] Betsch, P, editor. Energy-momentum integrators for elastic cosserat points, rigid bodies, and multibody systems. Structure-preserving Integrators in nonlinear structural dynamics and flexible multibody dynamics. (2016) Springer International Publishing.

[13] Gebhardt, C. G., Hofmeister, B., Hente, C. and Rolfes, R. Nonlinear dynamics of slender structures: a new object-oriented framework. Computational Mechanics. (2019) 63(2): 219-252.

[14] Orden, J. C. G. Energy and symmetry-preserving formulation of nonlinear constraints and potential forces in multibody dynamics. Nonlinear Dynamics. (2019) 95(1): 823-837. 